\title{
Correction to: Japanese catadromous fourspine sculpin, Rheopresbe kazika (Jordan \& Starks) (Pisces: Cottidae), transferred from the genus Cottus
}

\author{
Akira Goto • Ryota Yokoyama • Izumi Kinoshita • \\ Harumi Sakai
}

Published online: 17 January 2020

(C) Springer Nature B.V. 2020

\section{Correction to: Environ Biol Fish \\ https://doi.org/10.1007/s10641-019-00921-3}

The original version of this article unfortunately contained mistakes introduced during the production process. Tables 1 and 2 were presented incorrectly. The correct Tables are now given below:

The online version of the original article can be found at https://doi.org/10.1007/s10641-019-00921-3

\footnotetext{
A. Goto $(\bowtie)$

Department of Science of Environmental Education, Hokkaido University of Education, Hachiman-cho 1-2, Hakodate, Hokkaido 040-8567, Japan

e-mail: akir@ fish.hokudai.ac.jp

R. Yokoyama

Civil Engineering \& Eco-Technology Consultants, Co., Ltd, 2-23-2 Higashi-Ikebukuro, Tokyo, Toshima 170-0013, Japan

I. Kinoshita

USA Institute of Marine Biology, Kochi University, 194, Tosa,

Kochi 781-1164, Japan

H. Sakai

Department of Applied Aquabiology, National Fisheries University, 2-7-1 Nagata-honmachi, Shimonoseki, Yamaguchi 759-6595, Japan
} 
Table 1 Comparison of morphological characteristics in adult and larvae to juvenile stages among the fourspine sculpin Rheopresbe kazika, Cottus species and Trachidermus fasciatus

\begin{tabular}{|c|c|c|c|c|c|c|}
\hline & \multicolumn{3}{|l|}{ Adult stage } & \multicolumn{3}{|l|}{ Larval to juvenile stages } \\
\hline & Bony r. . $^{1)}$ & Villiform teeth $^{2)}$ & Preop. sp. ${ }^{3)}$ & Pariet. sp. ${ }^{4)}$ & Body depth & Melanoph. on p. f. ${ }^{5)}$ \\
\hline Rheopresbe kazika & absent & present & 4 & present & markedly deep & densely distribution \\
\hline Cottus species & absent & absent or rarely present & 1 to 3 & absent or rarely present & shallow & no distribution \\
\hline Trachidermus fasciatus & present & present & 4 & present & shallow & densely distribution \\
\hline
\end{tabular}

1) Bony ridge lines, 2) Villiform teeth on the palatines, 3) No. of Preopercular spines, 4) Parietal spines and 5) Melanophores on the pectoral fins. Data on adult morphology cited from Jordan and Starks (1904), Watanabe (1960), Nakamura (1963), Yabe (1985) and Fujii (2001). Data on larval and Juvenile morphology cited from Goto (1977), Takeshita et al. (1997), Harada et al. (1999), Kinoshita et al. (1999) and Kojima (2014)

Table 2 Karyotypes of Rheopresbe kazika, Trachidermus fasciatus and Cottus species

\begin{tabular}{|c|c|c|c|c|}
\hline Species & Biarmed chromosomes (M and SM) & Uniarmed chromosomes (A) & $2 \mathrm{~N}$ & NF \\
\hline Rheopresbe kazika ${ }^{1)}$ & 18 & 22 & 40 & 58 \\
\hline Trachidermus fasciatus ${ }^{1)}$ & 24 & 16 & 40 & 64 \\
\hline Cottus gobio ${ }^{3)}$ & 6 & 46 & 52 & 58 \\
\hline C. hangiongensis ${ }^{2)}$ & 6 & 42 & 48 & 54 \\
\hline C. nozawae $^{2)}$ & 10 & 38 & 48 & 58 \\
\hline C. poecilopus ${ }^{3)}$ & 8 & 40 & 48 & 56 \\
\hline C. pollux ${ }^{1)}$ & 10 & 38 & 48 & 58 \\
\hline C. reinii ${ }^{1)}$ & 12 & 36 & 48 & 60 \\
\hline
\end{tabular}

M: metacentrics, SM: submetacentrics, A: acrocentrics, 2N: number of chromosomes, NF: Total number of chromosome. Arm number cited, 1) from Abe (1972), 2) from Abe (1976), 3) from Starmach (1967)

The original article has been corrected. 